\title{
Efficacy and safety of erdosteine in the treatment of chronic rhinosinusitis with nasal polyposis - a pilot study*
}

\author{
Jiří Hoza ${ }^{1, \#, ~ R i c h a r d ~ S a l z m a n ~}{ }^{1, \#, ~ I v o ~ S t a ́ r e k ', ~ P e t r ~ S c h a l e k ², ~ R e n a t a ~ K e l l n e r o v a ́ 3 ~}$ \\ ' Department of Otorhinolaryngology and Head\&Neck Surgery, University Hospital Olomouc, Palacky University Olomouc, \\ Olomouc, Czech Republic \\ 2 Department of Otorhinolaryngology and Head\&Neck Surgery, Royal Vinohrady Teaching Hospital, Prague, Czech Republic \\ ${ }^{3}$ Medical Department, Medicom International, Itd., Brno, Czech Republic
}

Rhinology 51: 323-327, 2013

DOI:10.4193/Rhino13.039

*Received for publication:

April 8, 2013

Accepted: June 24, 2013

\section{Summary}

Background: Erdosteine was originally developed as a mucolytic agent. It is a multimechanism substance with anti-bacterial, anti-oxidant, and most importantly anti-inflammatory effects. Given similar mechanisms of action (suppression of cytokines, including tumor necrosis factor $\mathrm{a}$ ), it could become a reasonable alternative to currently used treatments with macrolides or steroids.

Objective: To assess efficacy and safety of erdosteine in the treatment of chronic rhinosinusitis with nasal polyposis (CRSwNP).

Methodology: A prospective non-interventional post-authorisation study comparing patients treated with erdosteine only or the combination of erdosteine and nasal corticosteroid spray for CRSWNP. The end-points were pre- and post-treatment changes in endoscopic score and subjective evaluation of CRSwNP related symptoms using a 22-item Sinonasal Outcome Test questionnaire. Patients underwent nasal endoscopy and filled the questionnaire before and after the treatment.

Results: No patient experienced any adverse effect during the study. A comparison of pre- and post-treatment endoscopic findings and questionnaire values revealed significant reduction in both patient groups, with a significantly better response in the erdosteine only group.

Conclusion: Based on this pilot study, erdosteine seems effective in the treatment of CRSWNP and might become a reasonable alternative to currently used medication. The therapeutical role of erdosteine needs to be further assessed.

Key words: erdosteine, chronic rhinosinusitis, mucolytics, nasal polyps, sinonasal outcome test questionnaire

\section{Introduction}

Chronic rhinosinusitis (CRS) is an inflammatory condition typically characterized by nasal obstruction, deteriorated olfaction, nasal discharge, and facial pain lasting longer than 12 weeks ${ }^{(1)}$. Corticosteroids are at present considered the drug of choice in the treatment of CRS. Topical steroid sprays are the mainstay of the treatment. The role of corticosteroids in CRS supposedly results from down-regulation of eosinophil function by directly reducing their activation and indirectly reducing the secretion of cytokines ${ }^{(2)}$.
Cytokines are peptides or glycoprotein molecules that act as inter-cellular signals in immune and inflammatory responses. Recent research has helped to define their function in the ethiopathogenesis of CRS (3). The accumulation of interleukins and tumor necrosis factor a (TNF-a) leads to proliferation of fibroblasts producing mediators attracting eosinophiles to submucosal tissue in CRS ${ }^{(4)}$.

The study of Wallwork et al. showed a clinical effect of macrolides with significant improvements in a 20 -item Sinonasal 
Outcome Test questionnaire score and nasal endoscopy in CRS

${ }^{(3)}$. Macrolides have undisputed effects on reduction of interleukins and TNFa in cystic fibrosis ${ }^{(5,6)}$ and asthma ${ }^{(7)}$ patients. The European Position Paper on Rhinosinusitis and Nasal Polyposis 2012 recommends the long-term therapy with macrolides as standard treatment in CRS without nasal polyposis ${ }^{(1)}$.

Erdosteine was originally developed as a mucolytic agent more than 15 years ago ${ }^{(8)}$. It is a multimechanism substance with known mucolytic, anti-bacterial, anti-oxidant and most importantly anti-inflammatory effects ${ }^{(9)}$. It has been shown that erdosteine significantly decreases serum levels of cytokines, including TNFa ${ }^{(8,10)}$.

Our extensive review of the literature failed to identify a single study looking at the role of erdosteine in chronic rhinosinusitis. Given similar mechanisms of action, erdosteine seems to be a reasonable alternative to currently used long-term treatment with macrolides or oral steroids that show numerous adverse effects. On the contrary, erdosteine is generally very well tolerated (11,12).

Here we present a prospective non-interventional post-authorisation study with the primary aim to assess efficacy and safety of erdosteine in the treatment of patients suffering from CRS with nasal polyposis. After subsequent analysis revealed erdosteine to be efficient and safe in all patients, we looked into the comparison between the groups receiving different treatments: erdosteine only and a combination of erdosteine with nasal corticosteroid spray. The end-points were pre- and post-treatment changes in endoscopic score and subjective evaluation of CRS related symptoms using a SNOT-22 questionnaire.

\section{Materials and methods}

\section{Patients and study design}

We included 60 patients ( 36 males, 24 females, median $52.5 \pm$ 12.7 years, range 20-75 years) seen with CRS with nasal polyposis at the Departments of Otorhinolaryngology and Head \& Neck Surgery of two institutions between March and December 2011. One half of the patients was treated in The University Hospital Olomouc and the other half in The Royal Vinohrady Teaching Hospital in Prague. The study was registered by the Czech State Institute for Drug Control under reference number 1103140000. After initial nasal endoscopy, the patients filled a validated Czech version of the 22-item Sinonasal Outcome Test questionnaire (SNOT-22). It comprises of 22 symptoms and quality of life related questions. Answers rated 0 (for no problem) to 5 (as bad as it can be) are summed for each question ${ }^{(13)}$. Nasal polyposis was endoscopically assessed using a Lindholdt staging system $(\text { Table } 1)^{(14)}$. The first group of 33 patients received solely erdosteine $300 \mathrm{mg}$ twice a day for the period of 3 months. We with-

held the standard CRS treatment during the course of erdosteine in this patient group. The recommended steroid treatment was then started in all patients with persistent findings. The second group of 27 patients received erdosteine in combination with nasal corticosteroid spray (mometasone furoate). All patients were re-assessed endoscopically and filled the SNOT-22 questionnaire again after the treatment was over. A difference between pre-treatment and post-treatment SNOT-22 mean values were used for further analysis. Two very experienced consultant rhinologists (a single surgeon at each institution) with special interest in endonasal surgery (having done over 1500 functional endonasal endoscopic procedures each) performed all initial and follow-up endoscopies at each institution.

\section{Statistical methods}

A paired Wilcoxon test was used to compare pre- and posttreatment values of SNOT-22 and endoscopic score in each group. The Kruskal-Wallis and Mann Whitney tests were used to compare the treatment effectiveness based on presence of allergy and between different stages of nasal polyposis. A p-value under 0.05 was considered significant. All statistical analyses were performed using the STATISTICA for Windows software (Statsoft Inc., Tulsa, OK, USA).

\section{Results}

\section{Demographics}

A comparison of all studied clinical and demographic parameters (sex, age, nasal polyposis stages, allergies) among both patient groups didn't reveal any significant differences ( $p>0.05$, Mann-Whitney), with an exception of pre-treatment endoscopic score on the right side $(p=0.02)$.

\section{Therapy safety}

None of the adverse drug reactions of $300 \mathrm{mg}$ erdosteine taken twice a day reported during the 3 months study were serious. Five of them (swelling of gums, heartburn, diarrhea, and 2 cases of nausea) were expected. The only unexpected adverse effect was mood disorder in one patient. However, the role of concomitant nasal corticosteroid administration cannot be excluded.

Table 1. Endoscopic staging system for nasal polyposis.

$0 \quad$ No polyps present

$1 \quad$ Polyps confined to middle meatus

2 Polyps beyond middle meatus (reaching inferior turbinate or medial to middle turbinate)

3 Polyps almost or completely obstructing nasal cavity 


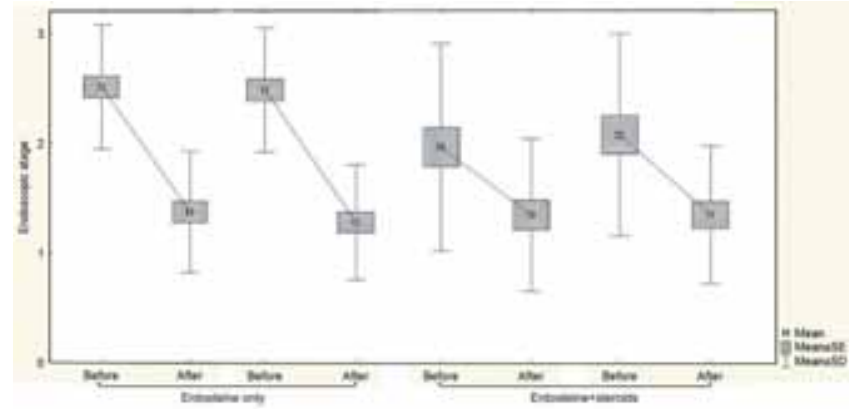

Figure 1. Change in endoscopic stage. The statistically significant drop of endoscopic stages from before to after treatment values in both studied patient groups (the values shown indicate pre-treatment endoscopic stage after deduction of post-treatment stage). The figure shows more significant reduction of nasal polyposis in the Erdosteine only group when compared with the patient group on combined treatment $(p<0.01$, Mann-Whitney). The right nostril is shown as vertically hatched and left one as horizontally hatched boxes.

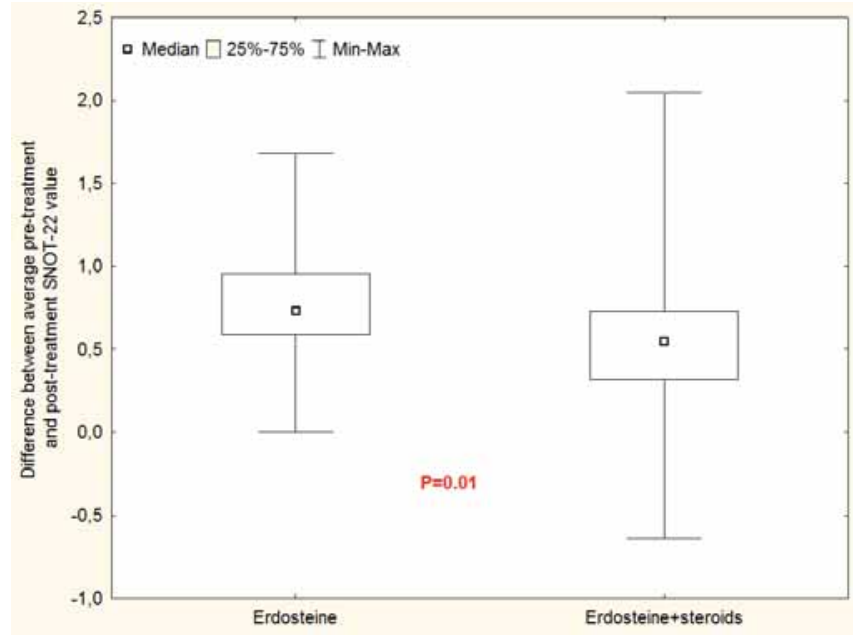

Figure 2. Change in SNOT-22 values. The Erdosteine only therapy was more efficient in improving patients' subjective symptoms when assessed by difference between averaged SNOT-22 values between before and after treatment ( $p=0.02$, Mann-Whitney).

Patients treated with erdosteine only

A comparison of endoscopic findings separately on each side revealed a significant reduction of nasal polyposis (bilaterally $p$ $<0.001$, Figure 1). Similarly, SNOT-22 values were significantly decreased after treatment ( $p<0.001$, all Wilcoxon, Figure 2$)$.

Patients treated with combination of erdosteine and nasal corticosteroid spray

This patient group showed good responses to treatment by decreased nasal polyposis stage (right $p<0.001$, left $p<0.001$, Figure 1). Also, the subjective evaluation of symptoms using

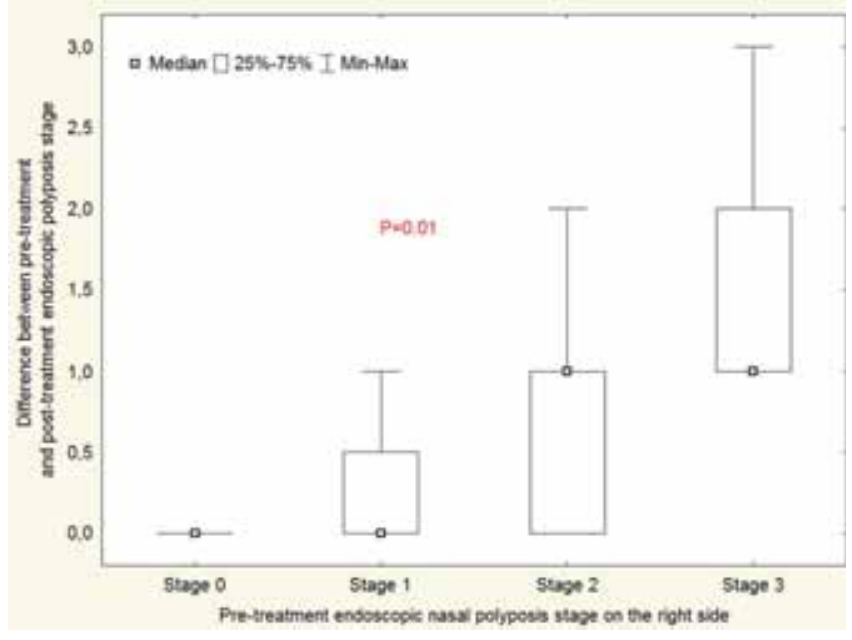

Figure 3. Response to treatment based on pre-treatment polyposis stage. The better response to treatment was more evident in higher pretreatment stages of nasal polyposis.

SNOT-22 improved significantly after treatment $(p<0.001$, all Wilcoxon, Figure 2).

\section{Comparison of therapy effectiveness between the patient groups}

The erdosteine only group benefited more from the treatment given than the group on combined treatment. A comparison of both endoscopic stage (right $p=0.002$, left $p=0.007$, Figure 1 ) and subjective evaluation based on SNOT-22 ( $p=0.02$, all MannWhitney, Figure 2) revealed a significant difference between the patient groups.

\section{Comparison of therapy effectiveness between different} stages of nasal polyposis

Higher stages of nasal polyposis before treatment responded more to therapy in both study groups. The comparison between different stages of nasal polyposis revealed a difference between pre- and post-treatment endoscopic grade and SNOT22 score (all $p<0.05$, right $R=0.62$, left $R=0.63$, Kruskal-Wallis, Figure 3 ). The therapy was most efficient in patients with the most advanced polyposis (stage 3 according to the Lindholdt classification) ( $p<0.05$, Mann-Whitney).

\section{Therapy effectiveness and allergy}

Among all 60 enrolled patients, 14 patients from the erdosteine only group, and 13 patients from the erdosteine + steroid group, suffered from allergies. Our study didn't show a different response (endoscopic stage and SNOT-22) to treatment based on presence of allergy ( $p>0.05$, all Mann-Whitney).

\section{Discussion}

CRS is a group of disorders characterized by inflammation of the 
mucosa of the nose and paranasal sinuses lasting longer than 12 weeks ${ }^{(1)}$. Chronic rhinosinusitis with and without nasal polyposis is distinguished. A postal survey suggested that $16 \%$ of the American adult population suffers from CRS ${ }^{(15)}$. The group of CRS disorders annually accounts for 18 million office visits in the USA ${ }^{(16)}$. National health care costs in the US are estimated at 8.6 billion dollar per year and health care spending is significantly greater in sinsusitis than in other chronic diseases such as ulcer disease, asthma and allergic rhinitis ${ }^{(1,17)}$. Therefore, improved treatment of CRS would reduce a large socio-economic burden on society ${ }^{(18)}$.

Corticosteroids are the mainstay of CRS treatment, down-regulating eosinophil function by directly inhibiting their activation and indirectly by decreasing cytokine secretion ${ }^{(2)}$. Production of cytokines was shown to be significantly suppressed in homogenates of nasal polyp tissue after treatment with prednisolone ${ }^{(3)}$. Lennard demonstrated a significant reduction of interleukin-5 expression in the mucosa of patients with chronic rhinosinusitis treated with systemic corticosteroids ${ }^{(4)}$. There is, also, evidence that similar effect is manifested after intranasal application of corticosteroids with limited systemic, but more profound topical effect directly on nasal mucosa ${ }^{(19)}$.

There has been growing interest in macrolides generally in treatment of airway inflammatory disease, including CRS, given by their not only anti-bacterial, but rather anti-inflammatory and immuno-modulatory effects ${ }^{(1)}$. Wallwark et al. concluded that the capacity of clarithromycin to inhibit the production of interleukins is responsible for the clinical efficacy of macrolide antibiotics in the treatment of chronic airway inflammation ${ }^{(3)}$.

Both corticosteroids and macrolides are associated with adverse effects, especially when used for a long time. The most common adverse effects of topical steroid treatment seems to be nose bleed, intra-nasal stinging, burning sensation, moderate pain, throat irritation, cough and dry skin ${ }^{(20,21)}$. Even though these symptoms seem minor, the lengthy application has significant impact on patients' quality of life. Adverse effects of long-term low-dose antibiotic treatment are mainly on bacterial resistance. Other less significant, but well recognized, adverse effects include gastrointestinal discomfort, skin rash, and elevation of liver enzyme levels ${ }^{(22)}$.

Erdosteine has been used in chronic inflammatory conditions of the lower respiratory tract. The patients on long-term treatment with erdosteine for chronic obstructive pulmonary disease had fewer acute exacerbations and improved quality of life ${ }^{(23)}$. Importantly, erdosteine is associated with a low incidence of adverse effects, most of which are gastrointestinal and generally very mild ${ }^{(11,12)}$. This was confirmed in our study. All monitored adverse drug reactions were not serious and confirmed good tolerability of the treatment.

We considered the significant difference in the pre-treatment stage of nasal polyposis between the patient groups without any clinical consequence as a good response to treatment given and was seen bilaterally in all studied groups.

Our study revealed that both study groups, i.e. the erdosteine only and the erdosteine + steroids groups, showed significant improvement in both local findings on nasal polyps as well as subjective evaluation of symptoms based on SNOT-22. Since this is the very first published study of erdosteine in CRS, there is no comparable literature available. We decided to re-assess the severity of CRS after 3 months, because we had treated only one group of patients with erdosteine, which is not listed as a recommended treatment for CRS. Had this novel treatment no effect, we didn't want to withhold steroid treatment for a longer period of time. We will address the appropriate duration of treatment in future studies. Should our results be confirmed by future trials against placebo, erdosteine could become a widely used treatment option in CRS.

The most important, although surprising, result is that the erdosteine only group showed significantly better results when compared with the group on combined treatment. So far, we cannot offer any justified explanation for this result. It seems that erdosteine has an antagonistic effect to that of corticosteroids. Further molecular biology studies need to be conducted to elucidate their interactions. We cannot rule out a selection bias causing better effects in erdosteine only patients as this study was non-interventional without proper randomization.

Comparing subgroups of patients with different endoscopic stages of nasal polyps before treatment indicated that higher stages responded significantly more than less advanced polyposis in both groups. This has not been reported previously in CRS patients treated with corticosteroids. However, in a histomorphological study, Bachert et al. observed a lower accumulation of eosinophils in early stage nasal polyps than in mature polyps ${ }^{(24)}$. We suspect that the higher number of receptive eosinophils in advanced nasal polyposis the more significant shrinkage due to the anti-interleukin effect of erdosteine. This again requires further studies.

Based on this pilot study, erdosteine might become a reasonable alternative to currently used medicaments for CRS therapy. The therapeutical role of erdosteine needs to be further assessed in a prepared randomized control trial against placebo, topical, and/or oral corticosteroid. Future studies should focus on the molecular biology of erdosteine action in the nasal mucosa. 


\section{Acknowledgements}

The study was supported by Medicom International, Itd., Czech Republic. The study was supported by the Institutional Support of Ministry of Health, Czech Republic, Nr. 1 RVO-FNOL2013.

\section{Authorship contribution}

$\mathrm{JH}$ : Performed surgeries, initiated the study, collected data, revised manuscript.
RS: Study design, statistical analysis, manuscript preparation IS: Study design, manuscript revision

PS: Performed surgeries

RK: Manuscript preparation and revision

\section{Conflict of interest}

None declared.

\section{References}

1. Fokkens WJ, Lund VJ, Mullol J, et al. European position paper on rhinosinusitis and nasal polyposis 2012. Rhinol Suppl. 2012; 23: 1-298

2. Roca-Ferrer J, Mullol J, Lopez E, et al. Effect of topical anti-inflammatory drugs on epithelial cell-induced eosinophil survival and GM-CSF secretion. Eur Respir J. 1997; 10: 1489-1495

3. Wallwork B, Coman W, Feron F, Mackay-Sim A, Cervin A. Clarithromycin and prednisolone inhibit cytokine production in chronic rhinosinusitis. Laryngoscope. 2002; 112: 1827-1830

4. Lennard CM, Mann EA, Sun LL, Chang AS, Bolger WE. Interleukin-1 $\beta$, Interleukin-5, Interleukin-6, Interleukin-8, and Tumor Necrosis Factor-a in chronic sinusitis: response to systemic corticosteroids. Am J Rhinol. 2000; 14: 367-373.

5. Equi AC, Davies JC, Painter $\mathrm{H}$, et al. Exploring the mechanisms of macrolides in cystic fibrosis. Respirat Med. 2006; 100: 687-697.

6. Wolter J, Seeney S, Bell S, Bowler S, Masel P, McCormack J. Effect of long term treatment with azithromycin on disease parameters in cystic fibrosis: a randomised trial. Thorax. 2002; 57: 212-216.

7. Kostadima E, Tsiodras S, Alexopoulos El, et al. Clarithromycin reduces the severity of bronchial hyperresponsiveness in patients with asthma. Eur Resp J. 2004; 23: 714-717.

8. Dal Negro RW, Visconti M, Micheletto C, Tognella S. Changes in blood ROS, e-NO, and some pro-inflammatory mediators in bronchial secretions following erdosteine or placebo: A controlled study in current smokers with mild COPD. Pulm Pharmacol Ther. 2007; 21: 304-308.

9. Dal Negro RW. Erdosteine: antitussive and anti-inflammatory effects. Lung. 2008; 186 : 70-73.

10. Jang YY, Song JO, Shin YK, Han ES, Chung
SL. Depressant effect of ambroxol and erdosteine on cytokine synthesis, granule enzyme release and free radical production in rat alveolar macrophages activated by lipopolysaccharide. Pharmycol Toxicol. 2003; 92: 173-179.

11. Moretti M. Pharmacology and clinical efficacy of erdosteine in chronic obstructive pulmonary disease. Expert Rev Resp Med. 2007; 1: 307-316.

12. Cazzola M, Floriani I, Page CP. The therapuetic efficacy of erdosteine in the treatment of chronic obstructive bronchitis: a meta-analysis of individual patient data. Pulm Pharmacol Thera. 2010; 23: 135-144.

13. Schalek P, Otruba L, Hahn A. Quality of life in patients with chronic rhinosinusitis: a validation of the Czech version of SNOT-22 questionnaire. Eur Arch Otorhinolaryngol. 2010; 267: 473-475.

14. Lildholdt T, Rundcrantz $H$, Lindqvist $N$. Efficacy of topical corticosteroid powder for nasal polyps: a double-blind, placebo-controlled study of budesonide. Clin Otolaryngol Allied Sci. 1995; 20: 26-30.

15. Blackwell DL, Collins JG, Coles R. Summary health statistics for U.S. adults: National Health Interview Survey, 1997. Vital Health Stat 10. 2002; 205: 1-109.

16. Anand VK. Epidemiology and economic impact of rhinosinusitis. Ann Otol Rhinol Laryngol. 2004; 193 (Suppl): S3-5.

17. Bhattacharyya N. Incremental health care utilization and expenditures for chronic rhinosinusitis in the United States. Ann Otol Rhinol Laryngol. 2011; 120: 423-427.

18. Fokkens WJ, Lund VJ, Mullol J. European position paper on rhinosinusitis and nasal polyps 2007. Rhinol Suppl 2007; 20: 1-136.

19. Parikh A, Scadding GK, Darby Y, Baker RC. Topical corticosteroids in chronic rhinosinusitis: a randomized, double-blind, placebo-controlled trial using fluticasone propionate aqueous nasal spray. Rhinology. 2001;
39: 75-79.

20. Poetker DM, Reh DD. A Comprehensive review of the adverse effects of systemic corticosteroids. Otolaryngol Clin North Am. 2010; 43: 753-768.

21. Periti P, Mazzei T, Mini E, Novelli A. Adverse effects of macrolide antibacterials. Drug Saf. 1993; 9: 346-364.

22. Seppala H, Klaukka T, Vuopio-Varkila J, et al. The effect of changes in the consumption of macrolide antibiotics on erythromycin resistance in group A streptococci in Finland. Finnish Study Group for Antimicrobial Resistance. N Engl J Med. 1997: 337: 441-446.

23. Moretti $M$, Bottrighi $P$, Dallari $R$, et al. The effect of long-term treatment with erdosteine on chronic obstructive pulmonary disease: the EQUALIFE Study. Drugs Exp Clin Res. 2004; 30: 143-152.

24. Bachert C, Gevaert P, Holtappels, G, Cuvelier $C$, van Cauwenberge P, Nasal polyposis: from cytokines to growth. Am J Rhinol. 2000; 14: 279-290.

\section{Richard Salzman \\ Department of Otorhinolaryngology \\ and Head \& Neck Surgery \\ University Hospital Olomouc \\ Palacky University Olomouc \\ I. P. Pavlova 6, 77520 Olomouc \\ Czech Republic}

Tel.: +420-588-444 186

Fax: +420-588-442 518

E-mail: richard.salzman@fnol.cz 